\title{
Characterizing the risk of melanoma and use of indoor tanning beds in high-risk groups to inform public health regulations
}

\author{
Casey Panning*, April Smith*, Olivia Spohn* and Leah van Wesenbeeck* \\ *Master of Public Health Program, University of Guelph, Guelph, ON. (All authors contributed equally to the paper.)
}

\begin{abstract}
There is concern about increasing incidence of melanoma and its association with indoor tanning devices. Overall, melanoma and indoor tanning devices exhibit a dose-response relationship; risk factors characterized for melanoma include age, gender, skin complexion, knowledge level, social norms, and location. Currently, legislative practices differ across Canada. Recommendations regarding intervention strategies, information dissemination, and legislation are discussed to inform public health regulations regarding the use of indoor tanning devices.
\end{abstract}

Key words: indoor tanning, artificial tanning, skin cancer, melanoma, risk factors, legislation

\section{Introduction}

There is growing concern about the incidence of various skin cancers, specifically melanoma, and its association with indoor tanning beds (Health Canada, 2014). Many studies have investigated the links between indoor tanning devices and the risk of melanoma. Various at-risk groups have been identified (Miyamoto et al., 2012; Guy et al., 2014). Our aim is to provide a comprehensive review of the characterization of melanoma and the use of tanning beds in high-risk groups. Finally, this evidence review will discuss current practices and future recommendations in terms of the use of indoor tanning devices, and the associated risk of melanoma.

\section{Methods}

The PubMed and MedLine databases were used to collect information regarding indoor tanning. Search terms used included "indoor tanning," "melanoma," "artificial tanning," and "tanning bed use". Only articles published between 2009 and 2014 were included in this review, because the World Health Organization changed its classification of indoor tanning devices as "carcinogenic to humans" in 2009 (Vogel et al., 2014). In addition, only English language studies conducted in the United States or Canada were included. Studies conducted outside of North America were excluded because the authors determined that research conducted in North America would

Corresponding authors: Casey Panning (email: panningc@ uoguelph.ca), April Smith (email: asmith54@uoguelph.ca), Olivia Spohn (email: ospohn@uoguelph.ca) or Leah Van Wesenbeeck (email: Ivanwese@uoguelph.ca) be more applicable to Canadian legislation. Research was compiled by first reviewing titles and abstracts, and then selecting relevant articles. To narrow the scope of the present review, focus was limited to the risk of melanoma associated with indoor tanning in high-risk populations.

\section{Melanoma and indoor tanning devices}

Several observational studies have characterized the risk of melanoma as it pertains to indoor tanning bed use. A casecontrol study conducted in Minnesota by Lazovich et al. (2010) looked at the risk of melanoma and exposure to tanning beds. They found indoor tanning use was reported in $63 \%$ of cases and $51 \%$ of controls. After adjusting for confounders, the risk of melanoma increased with higher frequency of indoor tanning bed use demonstrating a strong dose-response relationship (Lazovich et al., 2010).

More recently, Zhang et al. (2012) conducted a 20-year cohort study to collect information on the use of tanning beds in individuals throughout high school and college and between the ages of 25 and 35 years. Similar to Lazovich et al. (2010), Zhang et al. (2012) also found a dose-response relationship between tanning bed use and the combined risk of all skin cancers. They did not find significant risk associated with an increase in melanoma specifically as the researchers felt there were not enough melanoma cases for statistical power (Zhang et al., 2012).

An interesting connection linking tanning device use, the risk of melanoma, and individuals' DNA was characterized by Flores et al. (2013). They used a case-control study to look at how single-nucleotide polymorphisms (SNPs) can change the dopamine reward pathways in the brain, which would cause tanning 
to become a form of addiction. They found that some SNPs were associated with an increased likelihood of using indoor tanning beds, and SNPs in the same region of the brain were also associated with an increased risk for melanoma (Flores et al., 2013).

Finally, Vogel et al. (2014) tested the common perception that indoor tanning bed use can help protect against melanoma by preventing sunburns. Their evidence suggests that even when an individual does not burn (when tanning indoors or outdoors), the use of tanning beds still puts them at a greater risk for developing melanoma compared to with who do not use them at all (Vogel et al., 2014). Tanning is a response to DNA damage from UV radiation that precedes burning, and therefore is not protective (Vogel et al., 2014). In natural sunlight, a person is exposed to both UVA and UVB radiation. UVB radiation causes the majority of sunburns. Indoor tanning uses primarily UVA radiation; it allows individuals to tan for longer without burning, resulting in greater cumulative exposure (Zhang et al., 2012).

\section{Risk factors}

\section{Age}

The most prominent users of indoor tanning are those under the age of 25 years, and those at greatest risk are aged 17-21 years (Guy et al., 2014; Noar et al., 2014). It has been found that $30 \%-50 \%$ of female high school seniors and undergraduate students engage in indoor tanning (Stapleton et al., 2010; Guy et al., 2014). Using indoor tanning beds at a younger age leads to greater cumulative exposure, and therefore greater likelihood of developing melanoma (Lazovich et al., 2010). It has been found that teens may use indoor tanning beds to improve self-esteem (Guy et al., 2014), and this has been correlated with other "risky" health behaviours such as smoking, binge drinking, unhealthy weight management strategies, drug abuse, etc. (Guy et al., 2014; Simmons et al., 2014).

\section{Gender}

Females are consistently found to be the highest users of indoor tanning beds (Guy et al., 2014). Guy et al. (2014) suggested this finding may be related to the emphasis on the appearanceenhancement effects of indoor tanning. Forty-seven percent of female tanners engage in indoor tanning to feel more physically attractive and because they believe their social group is also engaging in indoor tanning (Stapleton et al., 2010). Indoor tanning among young females was also found to be associated with unhealthy weight control methods, binge drinking, abuse of recreational drugs, and unsafe sun practices (Guy et al., 2014). In males, use of indoor tanning was significantly related to nonprescribed steroid use, unhealthy weight management, and binge drinking (Miyamoto et al., 2012). Indoor tanning was significantly positively correlated with attempted suicide in males, whereas in female populations attempted suicide was negatively correlated with indoor tanning use (Miyamoto et al., 2012; Guy et al., 2014).

\section{Skin complexion}

Non-Hispanic, white individuals are the most frequent users of indoor tanning beds (Guy et al., 2014). It is argued those who are better able to tan, are at the greatest risk of utilizing indoor tanning (Lazovich et al., 2010; Vogel et al., 2014). They are likely to tan for longer and more frequently engage in outdoor tanning, resulting in greater cumulative exposure compared with those who burn easily (Lazovich et al., 2010; Vogel et al., 2014). Among melanoma patients who reported never having a sunburn, the odds of being an indoor tanner were four times higher among cases compared with controls (Vogel et al., 2014).

\section{Knowledge level}

The majority of indoor tanners in the high-risk groups were found to have above average knowledge surrounding safe sun practices (Stapleton et al., 2010; Guy et al., 2014; Simmons et al., 2014). It was hypothesized that the immediate effects of indoor tanning, such as feeling more physically attractive, superseded concerns about the harmful long-term implications of indoor tanning (Stapleton et al., 2010). Thirty-four percent of appearance-motivated indoor tanners were unaware that indoor tanning could increase their risk of melanoma (Stapleton et al., 2010).

\section{Social norms}

A number of studies found that social norms among friend groups and parents are fundamental in determining whether an individual will engage in indoor tanning (Stapleton et al., 2010; Noar et al., 2014; Simmons et al., 2014). Teens who perceived high rates of indoor tanning among their social groups and the media were more likely to engage in indoor tanning (Stapleton et al., 2010). Parental attitude towards tanning may influence an individual's decision to use indoor tanning. To illustrate, Utah minors can engage in indoor tanning with parental consent. Utah has experienced a smaller decline in indoor tanning compared with states that ban minors altogether, suggesting there are a number of parents who approve of indoor tanning (Simmons et al., 2014). It was also found that individuals whose parents had higher levels of education (i.e., were college graduates) were less likely to report indoor tanning, again suggesting that parental knowledge and attitudes influence young adults' decision to tan (Simmons et al., 2014).

\section{Location}

Indoor tanning beds are now accessible in tanning salons, fitness facilities, hair salons, spas, and some individuals have tanning beds in their homes (Ferrucci et al., 2014). While examining potential differences in UV radiation between indoor tanning at businesses compared with at one's home, it was discovered that virtually all artificial tanners engage in indoor tanning outside of the home (Ferrucci et al., 2014). Both tanning at home and at businesses were equally associated with an increased risk of melanoma, indicating that there is no "safe" place to tan (Ferrucci et al., 2014). Novel businesses offering tanning services may not be adequately training staff in tanning bed operations (Balaraman et al., 2013; Ferrucci 
et al., 2014). Businesses are not fully informing their customers of the risks of indoor tanning, and they are not adequately supervising tanning sessions (Balaraman et al., 2013; Ferrucci et al., 2014).

\section{Current practices}

\section{Canadian legislation}

Not all provinces across Canada have the same legislation with regards to tanning bed use among minors. Ontario, British Columbia, Quebec, and Prince Edward Island have banned youth under the age of 18 year from tanning bed use (British Columbia Ministry of Health, n.d.; Prince Edward Island Department of Health and Wellness, 2014; Quebec Legislative Assembly, n.d.). Nova Scotia, New Brunswick, and Newfoundland have banned youth under the age of 19 years (New Brunswick Legislative Assembly, 2013; New Foundland and Labrador Legislative Assembly, 2012; Nova Scotia Legislative Assembly, 2010). The remaining provinces do not have strict age regulations on tanning device use; Manitoba allows use under the age of 18 years with parental consent, and Alberta and Saskatchewan have no regulations against the use of tanning beds among minors (Manitoba Legislative Assembly, 2014).

Ontario passed the Skin Cancer Prevention Act (Tanning Beds) in 2013, which came into effect on 1 May 2014. The bill lays out specific guidelines on the sale and advertisement of commercial tanning beds in facilities across Ontario. Under this bill, businesses must disclose any commercial tanning beds on their property; must post signs specifying age restrictions and health implications associated with tanning bed use; and must request identification and provide protective eyewear for all customers using tanning facilities on their property (Ontario Legislative Assembly, 2013).

To reinforce this legislation, the Tanning Beds Compliance Protocol, 2014 provides information to local boards of health on how to monitor commercial tanning bed use and apply appropriate enforcement measures. Inspections are to be conducted if complaints regarding the facility's compliance with legislation are received by the board. Compliance with government of Ontario regulations will be enforced through inspection, education, and fines. Inspectors are to apply "progressive enforcement" to noncompliers; this is defined in the protocol as "the use of more stringent charging options to reflect the frequency and severity of the level of noncompliance." Fines can be up to $\$ 5,000$ if the noncomplier is an individual, and up to $\$ 25,000$ for businesses (Ontario Legislative Assembly, 2013). The other provinces with similar legislation instituted comparable practice regulations.

\section{Recommendations}

The present review has identified three areas of focus for future work to help address the growing incidence of melanoma related to tanning bed use. Researchers; federal, provincial, and local policy makers; and local public health units will be required to address the issues presented and develop an appropriate regulatory and educational solution. In combination, the following recommendations will address the major issues brought forth in this review.

\section{Intervention strategies}

Numerous reviewed studies emphasize the importance of further investigation into the motivations driving indoor tanning behaviour (Stapleton et al., 2010; Miyamoto et al., 2012; Guy et al., 2014). This was recommended to design effective, multifaceted, and comprehensive interventions (Miyamoto et al., 2012; Balaraman et al., 2013; Guy et al., 2014). Various studies have suggested the promotion of alternative behaviours such as sunless tanning or yoga, to address appearance or relaxation-based motivations (Roberts et al., 2009; Stapleton et al., 2010; Ontario Legislative Assembly, 2013; Guy et al., 2014). Interventions need to go beyond raising awareness of the skin damaging effects, as motivations regarding immediate appearance benefits are strong enough to cause individuals to disregard known risks (Stapleton et al., 2010). There is an identified need for further investigation into interventions that effectively target factors such as self-esteem, body image, and beliefs held by female adolescents regarding beauty (Stapleton et al., 2010; Guy et al., 2014). Such interventions have the potential to be more effective in promoting abstinence from indoor tanning (Stapleton et al., 2010; Guy et al., 2014). The health belief model is a common framework used to create behaviour change among targeted populations, and could be considered for use as a framework in the case of targeted indoor tanning intervention strategies.

\section{Information dissemination}

The reviewed studies highlight the need for consistent and accurate communication of known indoor tanning risks to the general public (Zhang et al., 2012; Balaraman et al., 2013; Guy et al., 2014; Vogel et al., 2014;). Information presented by tanning facilities needs to be monitored and regulated, especially regarding false health benefit claims (Lazovich et al., 2010; Zhang et al., 2012; Guy et al., 2014). It is similarly recommended that interventions focus on publicly discrediting these false claims of artificial tanning, instead of giving attention solely to associated risks (Noar et al., 2014). Because there is a growing number of novel locations offering indoor tanning services (e.g., fitness centres), there is a greater need for higher levels of training and education of employees regarding tanning risks (Balaraman et al., 2013; Ferrucci et al., 2014).

\section{Legislation implementation and enforcement}

Given the lack of consistent Canada-wide tanning bed legislature, it seems imperative to implement nation-wide guidelines as many studies recommend consistent, national age restrictions banning children under the age of 18 years from using tanning facilities (Zhang et al., 2012; Balaraman et al., 2013). Stricter regulations, enforcement policies, and increased surveillance of tanning facilities regarding procedure compliance are recommended (Simmons et al., 2014). To assess the impact of these different laws Simmons et al. (2014) noted the importance of looking at the difference in teen tanning bed use in jurisdictions where tanning beds are completely banned for minors compared with those that allow teens to tan if they have parental consent. Ferrucci et al. (2014) also recommended increased surveillance of novel tanning locations, such as gyms and apartment complexes, and they indicated the potential 
need of at-home tanning device regulations, as minors who are now unable to access tanning facilities may be utilizing athome resources. The implementation and enforcement of more stringent legislative restrictions may aid in reducing the use of tanning devices among minors, thus aiding in the prevention of melanoma (Roberts et al., 2009; Simmons et al., 2014). It is possible that a combination of both regulatory (e.g., legislature) and economic (e.g., financial sanctions) policy instruments could be used to achieve strict policy adherence.

\section{Gaps and limitations}

Identified gaps in the literature include information regarding effective intervention strategies (Noar et al., 2014). More information is required regarding tanner motivations, tanner subgroups, and intervention effectiveness. There is relatively little information about tanning devices used in novel facilities such as gyms, as this is a fairly new phenomenon. There were no Canadian specific investigations found regarding these factors, which could potentially limit the ability to effectively produce targeted interventions for Canadians. Our review focused on primary articles published between 2009 and 2014 in North America.

\section{Conclusion}

Overall, a dose-response relationship has been characterized between melanoma and the use of indoor tanning devices (Lazovich et al., 2010; Zhang et al., 2012). Youth are among the highest users of indoor tanning devices and, therefore, are at the highest risk for developing melanoma associated with use (Guy et al., 2014). There are several risk factors identified and characterized within our review. Legislation in combination with education should be used to restrict the use of indoor tanning devices among minors (Simmons et al., 2014). Tanning bed regulations in Canada currently vary by province. Although some provinces have established regulations, others lack any regulation at all, indicating a need for Canada-wide guidelines for use of tanning devices. Recommendations have been discussed regarding intervention strategies, information dissemination, and legislation to address the concern of increasing incidence of melanoma. This review has established the relationship between melanoma and tanning device use in high-risk groups to inform public health regulations regarding the use of indoor tanning devices.

\section{References}

Balaraman, B., Biesbroeck, L.K., Lickerman, S.H., Cornelius, L.A., and Jeffe, D.B. 2013. Practices of unregulated tanning facilities in Missouri: Implications for statewide legislation. Pediatrics, 131(3): 415-422. doi: 10.1542/peds.2012-1781.

British Columbia Ministry of Health, Government of British Columbia. n.d. B.C. ban on tanning bed use by youth under 18 . Available at http://www.health.gov.bc.ca/protect/tanning-bed-ban. html

Ferrucci, L.M., Isaksson Vogel, R., Cartmel, B., Lazovich, D., and Mayne, S.T. 2014. Indoor tanning in businesses and homes and risk of melanoma and nonmelanoma skin cancer in 2 US case-control studies. J. Am. Acad. Dertmatol, 71(5): 882-887. doi:10.1016/ j.jaad.2014.06.046.
Flores, K.G., Erdei, E., Luo, L., White, K.A., Leng, S., Berwick, M., et al. 2013. A pilot study of genetic variants in dopamine regulators with indoor tanning and melanoma. Exp. Dermatol, 22(9): 576581. doi:10.1111/exd.12200.

Guy, G.P., Berkowitz, A., Tai, E., Holman, D.M., Jones, S.E., and Richardson, L.C. 2014. Indoor tanning among high school students in the United States, 2009 and 2011. JAMA Dermatol, 150(5): 501-511. doi: 10.1001/jamadermatol.2013.7124.

Health Canada. Government of Canada announces stronger labelling requirements for tanning bedswarning labels remind users about skin cancer risk. 2014. Available at http://www.hc-sc.gc.ca/ahc-asc/ media/nr-cp/_2014/2014-025-eng.php. [accessed 30 November 2014].

Lazovich, D., Vogel, R.I., Berwick, M., Weinstock, M.A., Anderson, K.E., and Warshaw, E.M. 2010. Indoor tanning and risk of melanoma: A case-control study in a highly exposed population. Cancer Epidemiol Biomarkers Prev, 19(6): 1557-1568. doi: 10.1158/1055-9965.EPI-09-1249.

Manitoba, Legislative Assembly. 2014. The Public Health Amendment Act (Regulating Use of Tanning Equipment). Bill 225, 39th Legislature, 4th Session. (Assented June 17, 2010).

Miyamoto, J., Saraiya, M., and Berkowitz, Z. 2012. Indoor tanning device use among male high school students in the United States. J. Adolesc. Health., 50: 308-310. doi: 10.1016/j.jadohealth.2011.08.007.

New Brunswick, Legislative Assembly. 2013. Artificial Tanning Act. Bill 54, Chapter 2013, c.21. (Assented to June 21, 2013).

Newfoundland and Labrador, Legislative Assembly. 2012. An Act to Regulate the Personal Services Industry. Chapter P-7.2 (2012). St. John's: The Assembly, 2012. (Assented June 27, 2012).

Noar, S.M., Myrick, J.G., Morales-Pico, B., and Thomas, N.E. 2014. Development and validation of the comprehensive indoor tanning expectation scale. JAMA Dermatol, 150(5): 512-521. doi: 10.1001/jamadermatol.2013.9086.

Nova Scotia, Legislative Assembly. 2010. An Act to Regulate Tanning Beds. Bill 102, 61st Legislature, 2nd Session, 2010. Halifax: The Assemby. (Assented December 10, 2010).

Ontario Legislative Assembly. Skin Cancer Prevention Act (Tanning Beds), 2013. Bill 30, 40th Legislature, 2nd Session, 2013. Toronto: The Assembly. (Assented to Oct. 10, 2013).

Ontario Ministry of Health and Long-Term Care. 2014. Tanning beds compliance protocol, 2014. Toronto, ON: Queen's Printer for Ontario. Available at http://www.health.gov.on.ca/en/pro/programs/ publichealth/oph_standards/docs/tanning_beds_compliance.pdf [accessed 30 November 2014].

Prince Edward Island, Department of Health and Wellness, Government of Prince Edward Island. 2014. Tanning salons. Available at http://www.gov.pe.ca/health/index.php3?number $=$ 1040697 \&lang $=\mathrm{E}$.

Quebec, Legislative Assembly. An Act to Prevent Skin Cancer Caused by Artificial Tanning. Chapter C-5.2. Available at http://www2. publicationsduquebec.gouv.qc.ca/dynamicSearch/telecharge.php? type $=2 \&$ file $=/$ C_5_2/C5_2_A.html.

Roberts, D.J., Hornung, C.A., and Polk, H.C., Jr 2009. Another duel in the sun: Weighing the balances between sun protection, tanning beds, and malignant melanoma. Clin. Pediatr, 48(6): 614-622. doi: $10.1177 / 0009922809332589$.

Simmons, R., Smith, K., Balough, M., and Friedrichs, M. 2014. Decrease in self-reported tanning frequency among Utah teens following the passage of Utah senate bill 41: An analysis of the effects of youth-access restriction laws on tanning behaviors. J. Skin Cancer, 2014: 1-6. doi: 10.1080/14622200802239140. 
Stapleton, J., Turrisi, R., Hillhouse, J., Robinson, J.K., and Abar, B. 2010. A comparison of the efficacy of an appearance-focused skin cancer intervention within indoor tanner subgroups identified by latent profile analysis. J. Behav. Med, 33(3): 181-190. doi: 10.1007/s10865-009-9246-z.

Vogel, R., Ahmed, R., Nelson, H., Berwick, M., Weinstock, M., and Lazovich, D. 2014. Exposure to indoor tanning without burning and melanoma risk by sunburn history. J. Natl. Cancer Inst, 106(7): dju219. doi: 10.1093/jnci/dju219.

Zhang, M., Qureshi, A., Geller, A., Frazier, L., Hunter, D., and Han, J. 2012. Use of tanning beds and incidence of skin cancer. J. Clin. Oncol, 30(14): 1588-1593. doi: 10.1200/JCO.2011.39.3652. 